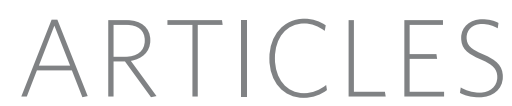

\title{
A Jurassic ceratosaur from China helps clarify avian digital homologies
}

Xing $\mathrm{Xu}^{1}$, James M. Clark ${ }^{2}$, Jinyou Mo ${ }^{3,4}$, Jonah Choiniere ${ }^{2}$, Catherine A. Forster ${ }^{2}$, Gregory M. Erickson ${ }^{5}$, David W. E. Hone ${ }^{1}$, Corwin Sullivan ${ }^{1}$, David A. Eberth ${ }^{6}$, Sterling Nesbitt ${ }^{7}$, Qi Zhao ${ }^{1}$, Rene Hernandez ${ }^{8}$, Cheng-kai Jia ${ }^{9}$, Feng-lu $\operatorname{Han}^{1,10}$ \& Yu Guo ${ }^{1,10}$

Theropods have traditionally been assumed to have lost manual digits from the lateral side inward, which differs from the bilateral reduction pattern seen in other tetrapod groups. This unusual reduction pattern is clearly present in basal theropods, and has also been inferred in non-avian tetanurans based on identification of their three digits as the medial ones of the hand (I-II-III). This contradicts the many developmental studies indicating II-III-IV identities for the three manual digits of the only extant tetanurans, the birds. Here we report a new basal ceratosaur from the Oxfordian stage of the Jurassic period of China (156-161 million years ago), representing the first known Asian ceratosaur and the only known beaked, herbivorous Jurassic theropod. Most significantly, this taxon possesses a strongly reduced manual digit I, documenting a complex pattern of digital reduction within the Theropoda. Comparisons among theropod hands show that the three manual digits of basal tetanurans are similar in many metacarpal features to digits II-III-IV, but in phalangeal features to digits I-II-III, of more basal theropods. Given II-III-IV identities in avians, the simplest interpretation is that these identities were shared by all tetanurans. The transition to tetanurans involved complex changes in the hand including a shift in digit identities, with ceratosaurs displaying an intermediate condition.

Ceratosaurs are suggested by many recent studies to be closely related to Tetanurae ${ }^{1,2}$, within which birds are nested, and they are mainly known from the Cretaceous southern hemisphere ${ }^{3-6}$. Our recent excavations in the Middle-Late Jurassic Shishugou Formation in the Junggar Basin of western China recovered a new ceratosaur, which is one of the earliest known ceratosaurs. This find sheds new light on the morphological evolution in Ceratosauria and in Theropoda as a whole and particularly the digital reduction pattern of theropods.

Theropoda Marsh, 1881

Ceratosauria Marsh, 1884

Limusaurus inextricabilis gen. et sp. nov.

Etymology. Limus, Latin for mud or mire; saurus, Latinization of Greek for lizard; inextricabilis, Latin for impossible to extricate. This name is in reference to the specimens' inferred death in a mire.

Holotype. Institute of Vertebrate Paleontology and Paleoanthropology (IVPP) V 15923 is an articulated, nearly complete skeleton (Fig. 1a, b).

Referred material. IVPP V 15924 is a semi-articulated skeleton missing the skull; it is 15\% larger than the holotype.

Locality and horizon. Wucaiwan area, Junggar Basin, Xinjiang; Oxfordian upper part of the Shishugou Formation ${ }^{7}$.

Diagnosis. Small ceratosaur with the following autapomorphies: short skull (half as long as the femur); skull and mandible toothless; nasal with a lateral shelf; premaxilla with a convex buccal edge; short and wide nasal less than one-third of skull roof length and only twice as long as wide; ventral process of lacrimal strongly inclined anteriorly; slender jugal with rod-like sub-orbital and sub-temporal rami; large external mandibular fenestra about $40 \%$ of mandibular length; flange on anterior margin of scapular blade; radius tightly adhering to ulna, and longer than the latter bone; olecranon process absent; metacarpal II much more robust than other metacarpals; metacarpal III with sub-triangular proximal articular surface and non-ginglymoidal distal end; metacarpal I highly reduced and carrying no phalanges; phalanx II-1 with distinct lateral process proximodorsally; pubis with laterally ridged, prominent posterior boot; metatarsus forming a strong transverse arch; robust ventral process at medial margin of proximal end of metatarsal III; metatarsal IV nearly straight, appressed against lateral surface of metatarsal III for nearly its whole length; and pedal digit I small, only $17 \%$ as long as metatarsal III.

\section{Morphological description and comparison}

Osteological and histological features indicate that both specimens of Limusaurus inextricabilis are young adults, probably between the exponential and stationary phases of growth (Fig. 1c; Supplementary Information). It shares some cranial features with both coelophysids and other ceratosaurs and also possess some unique features. Limusaurus has a fully developed rhamphotheca. Among non-avian theropods, this condition has been previously reported only in some Cretaceous coelurosaurs ${ }^{8}$, so this new find extends the distribution of rhamphothecae within theropods both temporally and phylogenetically.

Postcranially, Limusaurus displays a single, fused sternal plate. Unquestionable ossified sternal elements have been previously

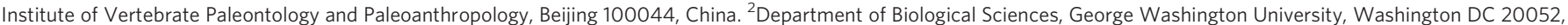
USA. ${ }^{3}$ Natural History Museum of Guangxi, Nanning, Guangxi 530012, China. ${ }^{4}$ Faculty of Earth Sciences, China University of Geosciences, Wuhan, Hubei 430074, China.

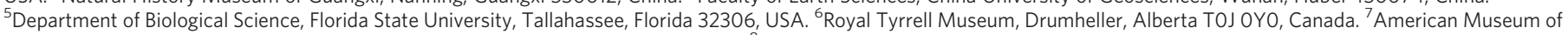

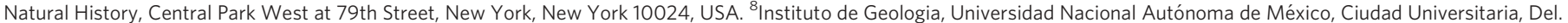

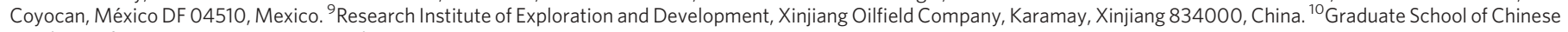
Academy of Sciences, Beijing 100039, China. 


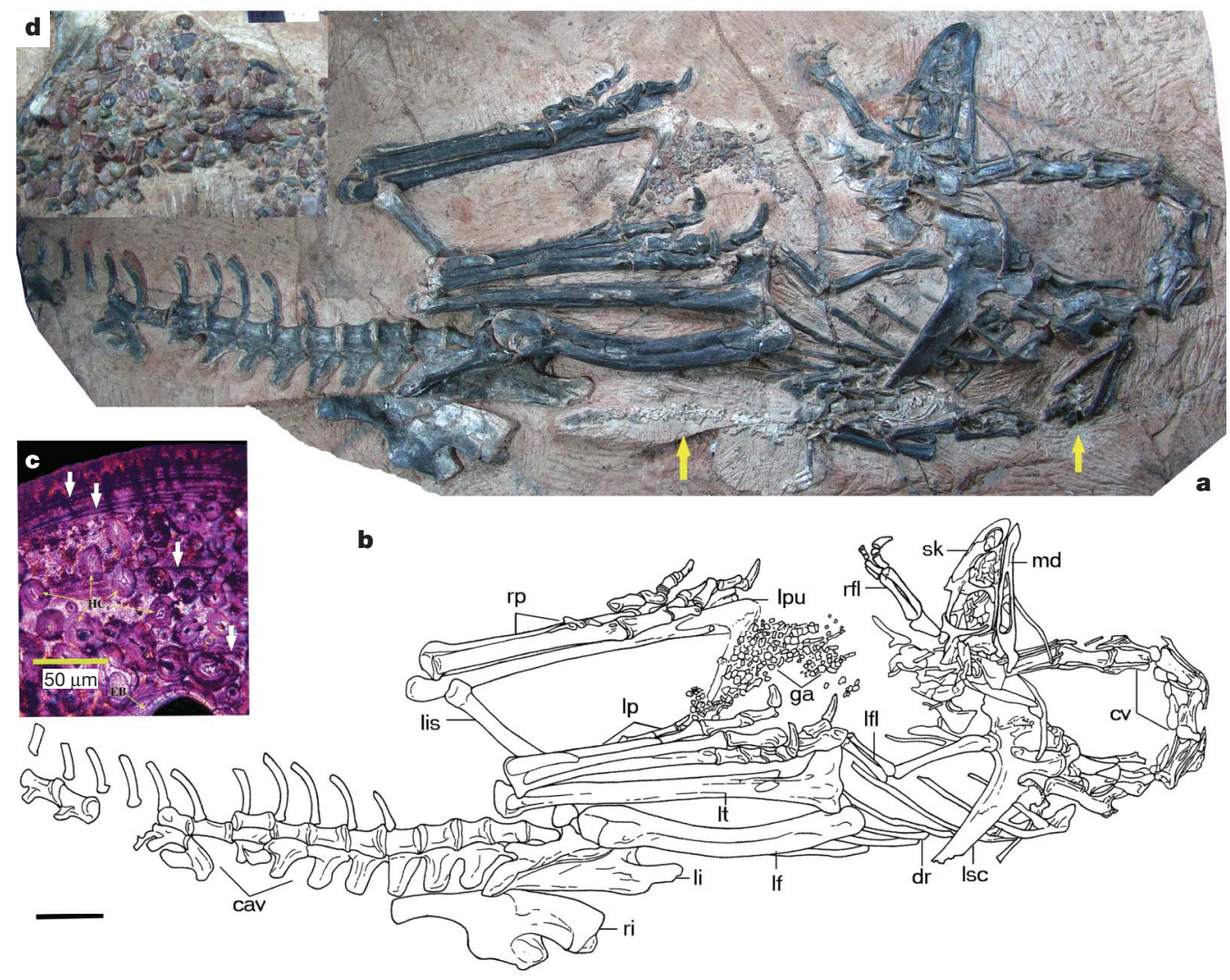

Figure 1 | Limusaurus inextricabilis (IVPP V 15923). Photograph (a) and line drawing (b) of IVPP V 15923. Arrows in a point to a nearly complete and fully articulated basal crocodyliform skeleton preserved next to IVPP V 15923 (scale bar, $5 \mathrm{~cm}$ ). c, Histological section from the fibular shaft of Limusaurus inextricabilis (IVPP V 15924) under polarized light. Arrows denote growth lines used to age the specimen; $\mathrm{HC}$ refers to round haversian canals and EB to layers of endosteal bone. The specimen is inferred to represent a five-year-old individual and to be at a young adult ontogenetic stage, based on a combination of histological features including narrower outermost zones, dense haversian bone, extensive and multiple endosteal bone depositional events and absence of an external fundamental system. d, Close up of the gastroliths (scale bar, $2 \mathrm{~cm}$ ). Abbreviations: cav, caudal vertebrae; cv, cervical vertebrae; dr, dorsal ribs; ga, gastroliths; If, left femur; lfl, left forelimb; li, left ilium; lis, left ischium; lp, left pes; lpu, left pubis; lsc, left scapulocoracoid; lt, left tibiotarsus; md, mandible; rfl, right forelimb; ri, right ilium; rp, right pes; sk, skull.

Biogeographically, Limusaurus is the first definitive ceratosaur known from East Asia ${ }^{4}$, to our knowledge, suggesting a cosmopolitan distribution for the group. In combination with other recent discoveries $^{13}$, this new ceratosaur makes the Asian dinosaurian fauna less endemic during the Middle-Late Jurassic, suggesting a faunal connection between Asia and other continents during that time period in spite of the presence of the Turgai Sea ${ }^{14}$.

\section{Convergent evolution of herbivory}

Limusaurus, ornithomimosaurs and shuvosaurid suchians such as Effigia are distantly related phylogenetically and also significantly separated temporally ${ }^{15,16}$, but they are remarkably similar in many gross skeletal features. They all have a small head with large orbits, toothless upper and lower jaws, a long neck and elongated hind limbs ${ }^{15}$. Limusaurus and shuvosaurids also share an extremely large mandibular fenestra and reduced forelimbs. Furthermore, both specimens of Limusaurus preserve gastroliths that are similar in quantity, size and shape (Fig. 1d) to those found in some ornithomimid specimens ${ }^{17}$. Another basal ceratosaur, Elaphrosaurus, was once actually placed within the Ornithomimosauria ${ }^{18}$. Together with the discovery of Limusaurus, this represents an extraordinary case of convergence among three higher archosaurian groups.

Some anatomical features of Limusaurus (small toothless head and long neck), and particularly the presence of a gastric mill, indicate a herbivorous diet. Secondary herbivory has previously been documented only in some relatively derived Cretaceous taxa among non-avian theropods ${ }^{17,19}$. As a basal ceratosaur from the Oxfordian, Limusaurus represents the earliest and most basal theropod inferred tetanurans are derived, thus providing further support for a close relationship between Ceratosauria and Tetanurae ${ }^{3}$. 


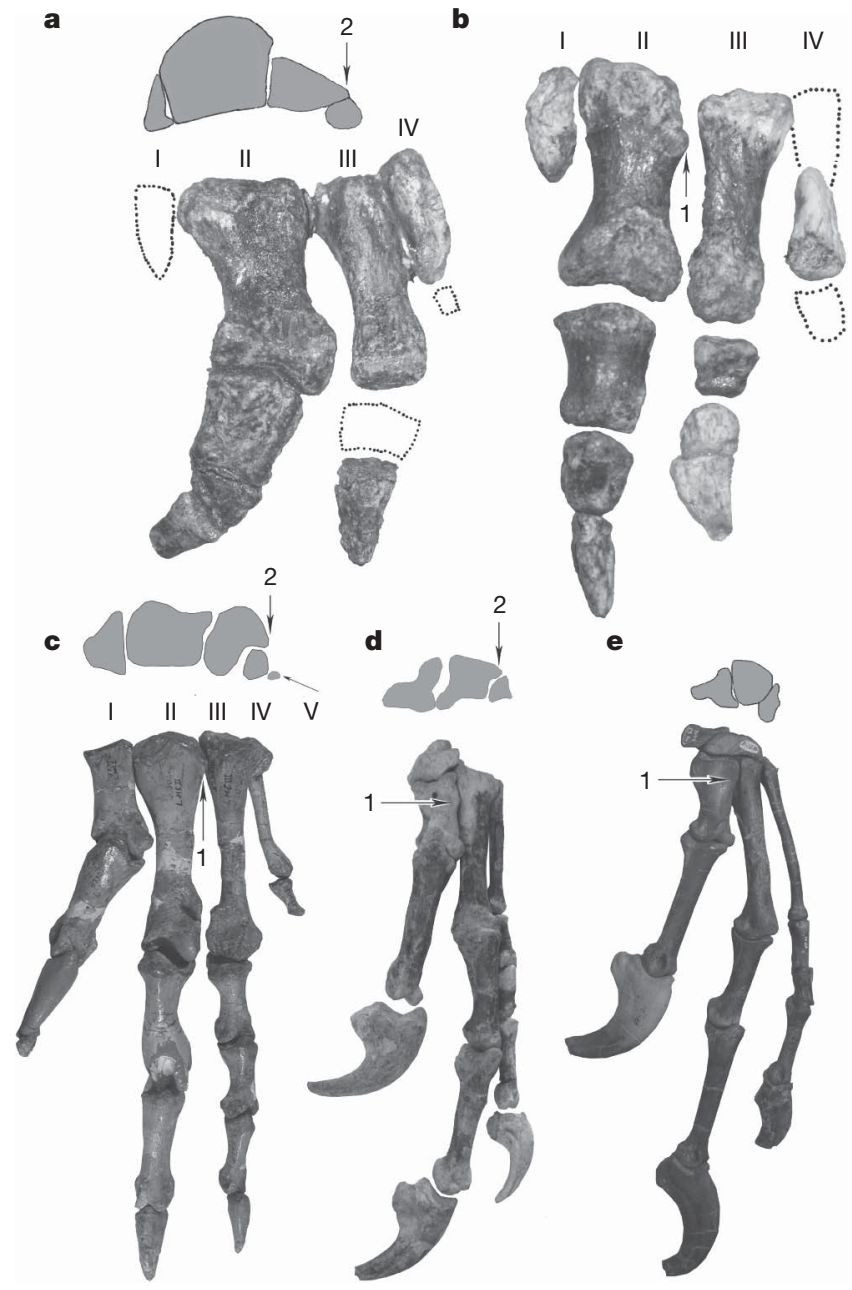

Figure 2 | Theropod manual morphologies as represented by several nonavian theropods. a, b, Ceratosaur Limusaurus (IVPP V 15923 and 15924); c, basal theropod Dilophosaurus (UCMP 37302); d, tyrannosauroid Guanlong (IVPP V14531); e, dromaeosaurid Deinonychus (YPM 5206). 1, dorsolateral process; 2 , metacarpal IV located ventral to metacarpal III. Note that the three metacarpals of Guanlong and Deinonychus display many similarities to metacarpals II-IV of Limusaurus and Dilophosaurus.

Interestingly, many metacarpal features, such as the contacts among the three metacarpals and the morphology of the lateral metacarpal, were previously considered to be tetanuran synapomorphies, but in fact can be better interpreted as retained unchanged from the condition in nontetanuran theropods if the three metacarpals of tetanurans are identified as II-III-IV.

to have been herbivorous, significantly expanding the known trophic diversity of Jurassic theropods.

\section{Manual digit reduction of theropods and avian digital homologies}

Theropods have long been assumed to display a pattern of lateral digit reduction (LDR), in which the digits have been progressively reduced from the lateral (that is, ulnar) side of the manus ${ }^{20-23}$. In contrast, bilateral digit reduction (BDR) is characteristic of most other tetrapod groups ${ }^{23}$. However, the reduction of digit I in Limusaurus constitutes strong new evidence for BDR in ceratosaurs, particularly because other ceratosaurs also possess a somewhat reduced digit $\mathrm{I}^{24,25}$.

The occurrence of BDR in Ceratosauria, the sister group of Tetanurae, invites a reconsideration of digital evolution in theropods as a whole, and particularly of the complex issue of tetanuran digital homologies ${ }^{21-23}$. On the basis of morphological evidence from fossil taxa, the three digits retained by tetanurans have traditionally been interpreted as homologues of digits I-III of the primitive theropod manus, exemplifying LDR $^{20,21}$. However, the discovery of BDR in Limusaurus and its close relatives introduces the possibility that this pattern might be more broadly distributed among non-avian theropods and indicates that the three digits of extinct tetanuran theropods could be II-IV, an alternative hypothesis previously little considered in palaeontological literature ${ }^{26}$.

Positional relationships have been widely accepted as the main operational criterion for primary homology ${ }^{27}$, although cases of positional shifts have been documented ${ }^{28}$ or experimentally induced ${ }^{29}$. In the present case, the conservative pentadactyl pattern seen in the embryos of extant birds and crocodilians, and by inference all crown-group archosaurs including theropods, provides a reliable reference system for topologically assessing the primary homologies of tetanuran digits. Recent developmental studies favour the II-III-IV hypothesis by showing that the three digits of the only living tetanurans, the birds, originate developmentally from the middle three of the five digital primordia ${ }^{23,30-33}$. Ontogenetic research on expression patterns of posterior Hoxd genes shows that digits that develop from positions II-III-IV in birds acquire a I-II-III identity later in ontogen $^{34,35}$, resolving the apparent conflict between palaeontological and developmental data. Despite the strength of this evidence, developmental data from extant taxa cannot indicate the point at which digital identities shifted during the evolution of the Theropoda, nor reveal the tempo of that shift. The fossil record remains the only source of information on these aspects of the transition. Furthermore, in fossil tetanurans early embryonic stages cannot be observed, so only morphological criteria are available to infer digit identities. Comparing the digits of tetanurans to those of their closest relatives, Ceratosauria and Dilophosaurus, is particularly helpful in elucidating digital primary homologies.

The main morphological features cited in support of the traditional I-II-III hypothesis include the topographic relationship of the 'semilunate' carpal to the metacarpus, the short and distally asymmetrical medial metacarpal, and the apparently conserved phalangeal formula of 2-3-4 (refs 20-22, 36). The phalangeal formula is particularly striking because it characterizes digits I-II-III across a wide range of disparate tetrapod groups ${ }^{21}$. However, contrary evidence can be adduced against each of these points. Theropod carpal homologies are complicated by anomalies such as the presence of a relatively small, separate medial distal carpal in non-maniraptoran tetanurans and the absence of a large distal carpal in Dilophosaurus ${ }^{37}$. Metacarpal II is distally asymmetric in Limusaurus, Dilophosaurus ${ }^{38}$ and some specimens of the coelophysid Coelophysis ${ }^{39}$, so the asymmetry of metacarpal $\mathrm{I}$ is not a unique feature. Finally, digits I-III do not display a 2-3-4 phalangeal formula in any known ceratosaur, demonstrating that the conservatism of this formula is not absolute.

New information from Limusaurus and various other theropods reveals a number of morphological features that support the alternative II-III-IV hypothesis (Fig. 2). In basal theropods the proximal ends of metacarpals I and II are mutually appressed without overlap whereas the dorsolateral corner of the proximal end of metacarpal II forms a flange that slightly overlaps the dorsal surface of metacarpal III. In Limusaurus and tetanurans, a similar flange extends distally to form a large, oblique contact between metacarpals II and III in Limusaurus and between the medial and middle metacarpals of tetanurans. This indicates that the medial and middle metacarpals of tetanurans correspond to metacarpals II and III. Similarly, the proximal end of metacarpal IV is appressed to the ventrolateral face of metacarpal III in non-tetanuran theropods. The lateral metacarpal of tetanurans contacts the ventrolateral face of the middle metacarpal in the same way, reinforcing the II-III-IV interpretation. The medial metacarpal of basal tetanurans is the most robust in the manus, like metacarpal II in more basal theropods including ceratosaurs, Dilophosaurus $^{38}$ and coelophysids ${ }^{3}$. The elongate proximal phalanx of the medial digit of tetanurans is similar to phalanx I-1 in some coelophysids but unlike the relatively short phalanx I-1 seen in 
ceratosaurs $^{3}$, Dilophosaurus ${ }^{38}$ and Herrerasaurus $^{40}$. The middle metacarpal is longer than the others in tetanurans, like metacarpal III in Limusaurus, Dilophosaurus ${ }^{38}$, at least some coelophysids, Herrerasaurus $^{40}$ and most other archosaurs ${ }^{26}$. The four-fingered ornithischian Psittacosaurus displays LDR, and its metacarpal III is also the longest in the manus ${ }^{41}$. A dorsolateral process is present on the proximal end of the middle metacarpal of basal tetanurans, and a similar process characterizes metacarpal III of Limusaurus, Dilophosaurus and Herrerasaurus. Finally, the lateral metacarpal is short, slender and proximally sub-triangular in outline in basal tetanurans, like metacarpal IV in non-tetanuran theropods ${ }^{3}$. It is noteworthy that most of these similarities are more evident between basal tetanurans and their close outgroups, Dilophosaurus and the ceratosaurs, than between derived tetanurans and coelophysids.

We conducted a quantitative analysis of digital homologies to test the alternative I-II-III and II-III-IV hypotheses in tetanurans (Supplementary Information). When birds are coded as II-III-IV, coding all Tetanurae as II-III-IV $(-\mathrm{V})$ is a minimum of ten steps shorter than a shift from I-II-III to II-III-IV anywhere within the Tetanurae, and four steps shorter than a shift at the base of the Averostra. Coding all Tetanurae as having II-III-IV is the same in tree length as (characters unordered) or six steps longer than (characters ordered) an alternative scheme in which all Tetanurae, including birds, are interpreted as having I-II-III, a hypothesis that clearly contradicts developmental data from extant birds. We conclude that, if birds possess digits II-III-IV as most developmental studies indicate, the data strongly support the interpretation that all tetanurans have digits II-III-IV $(-\mathrm{V})$, as outlined above. If extant birds are ultimately found to possess digits I-II-III, of course, then no conflict between neontological and traditional palaeontological data exists.

This implies the reduction of digit I before the divergence of the Ceratosauria and the Tetanurae, the appearance of some pollex-like features in digit II and the acquisition of a novel phalangeal formula (X-2-3-4-X) early in tetanuran evolution. Both modifications are partially indicated by the manual morphologies of ceratosaurs and more basal theropods. Also, they are indirectly supported by observations in living animals that a digit will display features normally associated with the neighbouring medial digit if the latter fails to chondrify in early development ${ }^{21}$, that phalangeal counts can vary even within species ${ }^{29,42}$ and that secondarily cartilaginous elements can regain their ability to ossify ${ }^{43}$.

The frameshift hypothesis of digital evolution in theropods holds that each digit, positionally defined, assumed morphological features that primitively characterized the next most medial digit due to homeotic transformations ${ }^{21}$. Recent studies indeed confirm that a homeotic change has affected the development of the avian digits: in extant birds, condensation II receives a Hox signal (absence of posterior Hoxd expression) appropriate for digit $\mathrm{I}^{34,35}$. The frameshift was, however, not necessarily a sudden, discrete event, and its repatterning of the digits was not complete, because the metacarpals of basal tetanurans in general retained key features indicating their original identities. The uneven modification of the tetanuran manus may reflect the fact that tetrapod digits develop from proximal to distal ${ }^{44}$, each metacarpal appearing before its associated phalanges. The manus may have been repatterned by a late-acting developmental signal that influenced the phalanges to a greater degree than the metacarpals.

In conclusion, both the I-II-III and II-III-IV hypotheses can draw some supporting morphological evidence from the hands of extinct tetanurans, but largely from different manual regions (Fig. 3). If extant tetanurans have retained the middle three digits, as many developmental studies suggest ${ }^{23,30-33}$, it is more parsimonious to identify the three digits of extinct tetanurans as digits II-IV. This new evidence from Limusaurus and other basal theropods suggests that a gradual homeotic shift in digit identity characterized early stages of theropod evolution, that an intermediate stage of this shift

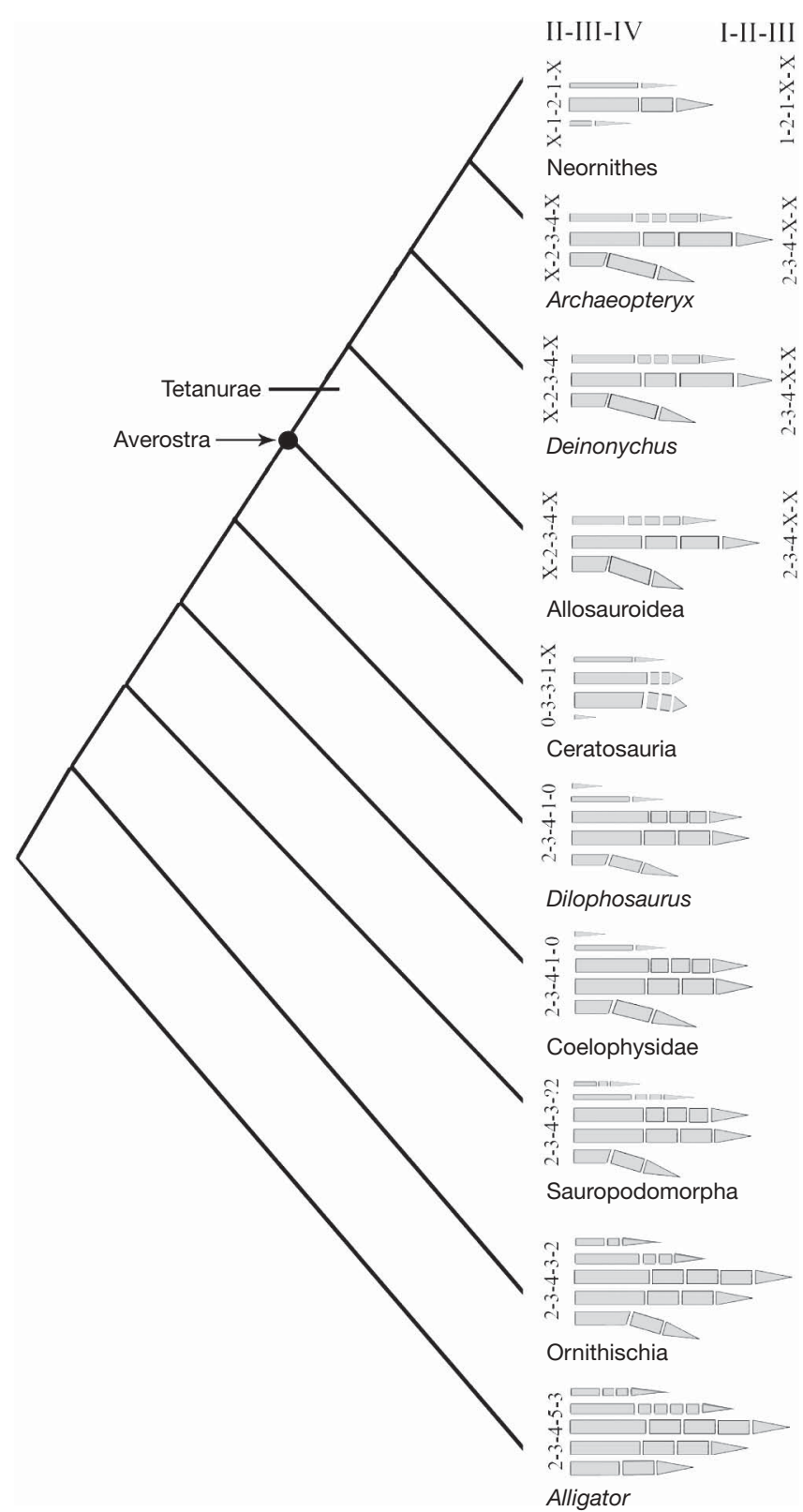

Figure 3 | Manual digital evolution in theropod dinosaurs. Manual digital evolution involves both BDR and LDR in theropod dinosaurs. The shift to BDR in ceratosaurs is coincident with features indicating a reduction in the grasping function of the manus. In ceratosaurs, the manus is small, the manual phalanges are abbreviated and the claws are non-raptorial. This supports the hypothesis that a grasping function constrained the hand to LDR in non-tetanuran theropods ${ }^{21}$. If BDR applies to the more inclusive Averostra, as the II-III-IV hypothesis suggests, early stages of tetanuran evolution must have involved loss of the already highly reduced metacarpal I, reduction in the length of metacarpal II and the reappearance of additional phalanges on metacarpal IV. Both the I-II-III and II-III-IV hypotheses can claim a degree of support from morphological data, but the II-III-IV hypothesis is more parsimonious when developmental data from extant birds are considered.

is preserved in the Ceratosauria and that the shift was complete by the time of the diversification of the earliest tetanurans.

Received 24 January; accepted 29 April 2009.

1. Rauhut, O. W. M. The Interrelationships and Evolution of Basal Theropod Dinosaurs (Palaeontological Association, 2003).

2. Carrano, M. T., Sampson, S. D. \& Forster, C. A. The osteology of Masiakasaurus knopfleri, a small abelisauroid (Dinosauria: Theropoda) from the Late Cretaceous of Madagascar. J. Vertebr. Paleontol. 22, 510-534 (2002). 
3. Tykoski, R. S. \& Rowe, T. in The Dinosauria 2nd edn (eds Weishampel, D. B. Dodson, P. \& Osmolska, H.) 47-70 (Univ. California Press, 2004).

4. Allain, R. et al. An abelisauroid (Dinosauria: Theropoda) from the Early Jurassic of the High Atlas mountains, Morocco, and the radiation of ceratosaurs. J. Vertebr. Paleontol. 27, 610-624 (2007).

5. Mateus, O., Walen, A.\& Antunes, M. T. The large theropod fauna of the Lourinhã Formation (Portugal) and its similarity to that of the Morrison Formation, with a description of a new species of Allosaurus. New Mexico Mus. Nat. Hist. Sci. Bull. 36, 123-129 (2006).

6. Carrano, M. T. \& Sampson, S. D. The phylogeny of Ceratosauria (Dinosauria: Theropoda). J. Sys. Palaeontol. 6, 183-236 (2008).

7. Eberth, D. A. et al. Sequence stratigraphy, paleoclimate patterns and vertebrate fossil preservation in Jurassic-Cretaceous strata of the Junggar Basin, Xinjiang Autonomous Region, People's Republic China. Can. J. Earth Sci. 38, 1627-1644 (2001).

8. Chiappe, L. M., Ji, S.-A., Ji, Q. \& Norell, M. A. Anatomy and systematics of the Confuciusornithidae (Theropoda: Aves) from the late Mesozoic of northeastern China. Bull. Am. Mus. 242, 1-89 (1999).

9. Holtz, T. R. J., Molnar, R. E. \& Currie, P. J. in The Dinosauria 2nd edn (eds Weishampel, D. B., Dodson, P. \& Osmolska, H.) 71-110 (Univ. California Press, 2004).

10. Christiansen, P. \& Bonde, N. Limb proportions and avian terrestrial locomotion. J. Ornithol. 143, 356-371 (2002).

11. Bonaparte, J. F. The Gondwanian theropod families Abelisauridae and Noasauridae. Hist. Biol. 5, 1-25 (1991).

12. Sereno, P. C. et al. Predatory dinosaurs from the Sahara and Late Cretaceous faunal differentiation. Science 272, 986-991 (1996).

13. $\mathrm{Xu}, \mathrm{X}$. et al. A basal tyrannosauroid dinosaur from the Late Jurassic of China. Nature 439, 715-718 (2006)

14. Russell, D. A. The role of Central Asia in dinosaurian biogeography. Can. J. Earth Sci. 30, 2002-2012 (1993)

15. Makovicky, P. J., Kobayashi, Y. \& Currie, P. J. in The Dinosauria 2nd edn (eds Weishampel, D. B., Dodson, P. \& Osmolska, H.) 137-150 (Univ. California Press, 2004).

16. Nesbitt, S. The anatomy of Effigia okeeffeae (Archosauria, Suchia), theropod-like convergence, and the distribution of related taxa. Bull. Am. Mus. Nat. Hist. 302, 1-84 (2007).

17. Kobayashi, Y. et al. Herbivorous diet in an ornithomimid dinosaur. Nature 402, 480-481 (1999).

18. Galton, P. M. Elaphrosaurus, an ornithomimid dinosaur from the Upper Jurassic of North America and Africa. Palaeontologische Zeitschrift 56, 265-276 (1982).

19. Xu, X., Cheng, Y. N., Wang, X. L., Chang, C. H. \& Chang, H. An unusual oviraptorosaurian dinosaur from China. Nature 419, 291-293 (2002).

20. Gauthier, J. in The Origin of Birds and the Evolution of Flight (ed. Padian, K.) 1-55 (California Academy of Sciences, 1986).

21. Wagner, G. P. \& Gauthier, J. A. 1,2,3 = 2,3,4: a solution to the problem of the homology of the digits in the avian hand. Proc. Natl Acad. Sci. USA 96, 5111-5116 (1999).

22. Shubin, N. H. in Homology: The Hierarchical Basis of Comparative Biology (ed. Hall, B. K.) 249-271 (Academic, 1994).

23. Burke, A. C. \& Feduccia, A. Developmental patterns and the identification of homologies in the avian hand. Science 278, 666-668 (1997).

24. Burch, S. \& Carrano, M. Abelisaurid forelimb evolution: new evidence from Majungasaurus crenatissimus (Abelisauridae: Theropoda). J. Vertebr. Paleontol. 28 (supplement to 3) 58A (2008).

25. Cortia, R. A., Chiappe, L. M. \& Dingus, L. A new close relative of Carnotaurus sastrei Bonaparte 1985 (Theropoda: Abelisauridae) from the Late Cretaceous of Patagonia. J. Vertebr. Paleontol. 22, 460-465 (2002).

26. Thulborn, R. A. \& Hamley, T. L. The reptilian relationships of Archaeopteryx. Aust. J. Zool. 30, 611-634 (1982).

27. Riedl, R. Die Ordnung des Lebendigen. Systembedingungen der Evolution (Parey, 1975).
28. Burke, A. C., Nelson, C. E., Morgan, B. A. \& Tabin, C. Hox genes and the evolution of vertebrate axial morphology. Development 121, 333-346 (1995).

29. Dahn, R. D. \& Fallon, J. F. Interdigital regulation of digit identity and homeotic transformation by modulated BMP signaling. Science 289, 438-441 (2000).

30. Larsson, H. C. E. \& Wagner, G. P. Pentadactyl ground state of the avian wing. J. Exp. Zool. B 294, 146-151 (2002).

31. Feduccia, A. \& Nowicki, Z. The hand of birds revealed by early ostrich embryos. Naturwissenschaften 89, 391-393 (2002).

32. Welten, M. C. Verbeek, F. J., Meijer, A. H. \& Richardson, M. K. Gene expression and digit homology in the chicken embryo wing. Evol. Dev. 7, 18-28 (2005).

33. Kundrát, M., Seichert, V., Russell, A. P. \& Smetana, K. Pentadactyl pattern of the avian wing autopodium and pyramid reduction hypothesis. J. Exp. Zool. B 294, 152-159 (2002).

34. Vargas, A. O. \& Fallon, J. F. Birds have dinosaur wings: the molecular evidence. J. Exp. Zool. B 304, 86-90 (2005)

35. Vargas, A. O., Kohlsdorf, T., Fallon, J. F., Brooks, J. V. \& Wagner, G. P. The evolution of HoxD-11 expression in the bird wing: insights from Alligator mississippiensis. PLoS ONE 3, e3325 (2008).

36. Chatterjee, S. Counting the fingers of birds and dinosaurs. Science 280, 355 (1998).

37. Chure, D. J. in New Perspectives on the Origin and Early Evolution of Birds (eds Gauthier, J. A. \& Gall, L. F.) 122-130 (Yale Univ. Press, 2001).

38. Welles, S. P. Dilophosaurus wetherilli (Dinosauria, Theropoda), osteology and comparisons. Palaeontgr. Abt. A 185, 85-180 (1984).

39. Galton, P. M. Manus movements of the coelurosaurian dinosaur Syntarsus and opposability of the theropod hallux. Arnoldia (Rhodesia) 5, 1-8 (1971).

40. Sereno, P. C. The pectoral girdle and forelimb of the basal theropod Herrerasaurus ischigualastensis. J. Vertebr. Paleontol. 13, 425-450 (1993).

41. You, H. L. \& Dodson, P. in The Dinosauria (eds Weishampel, D. B., Dodson, P. \& Osmolska, H.) 478-493 (Univ. California Press, 2004).

42. Drossopoulou, G. et al. A model for anteroposterior patterning of the vertebrate limb based on sequential long- and short-range Shh signalling and Bmp signalling. Development 127, 1337-1348 (2000).

43. Baksh, D., Boland, G. M. \& Tuan, R. S. Cross-talk between Wnt signaling parthways in human mesenchymal stem cells leads to functional antagonism during osteogenic differentiation. J. Cell. Biochem. 101, 1109-1124 (2007).

44. Shapiro, M. D. Developmental morphology of limb reduction in Hemiergis (Squamata: Scincidae): chondrogenesis, osteogenesis, and heterochrony. J. Morphol. 254, 211-231 (2002).

Supplementary Information is linked to the online version of the paper at www.nature.com/nature.

Acknowledgements The authors thank H.-J. Wang for organizing the fieldwork, R. S. Li for illustrations, L.-S. Xiang and X.-Q. Ding for preparing the specimens, X.-Q. Ding for editing the illustrations, M. Kundrát and J. Gauthier for critical comments, O. Rauhut, P. Makovicky and D. Chure for some theropod images, R.-S. Tykoski for references, and members of the Sino-American expedition team for collecting the fossil. The field work was supported by the National Natural Science Foundation of China, the National Science Foundation Division of Earth Sciences of the USA, the Chinese Academy of Sciences, the National Geographic Society, the Jurassic Foundation, the Hilmar Sallee bequest and George Washington University. Study of the specimens was supported by the Chinese Academy of Sciences, the National Science Foundation Division of Earth Sciences of the USA and the National Natural Science Foundation of China.

Author Contributions X.X. and J.M.C. designed the project. X.X., J.M.C., J.C., G.M.E., S. N. and J.-Y.M. performed the research. X.X., J.M.C., G.M.E., J.C., C.S. and D.W.E.H. wrote the manuscript. X.X., J.M.C., J.-Y.M., J.C., C.A.F., D.A.E., Q.Z., R. H., C.-K. J., F.-L.H. and Y.G. excavated the specimens.

Author Information Reprints and permissions information is available at www.nature.com/reprints. Correspondence and requests for materials should be addressed to X.X. (xingxu@vip.sina.com). 